\title{
Recurrent Cerebral Venous Thrombosis Associated with Elevated Factor VIII
}

\author{
Mi Jung Kim, M.D., A-Hyun Cho, M.D., Young-Joo No, M.D., Hee-Young Kim, M.D., \\ Jong S. Kim, M.D. \\ Department of Neurology, University of Ulsan College of Medicine, Asan Medical Center
}

\begin{abstract}
Cerebral venous thrombosis (CVT) rarely recurs, and the factors associated with a recurrence remain unclear. Recently, however, elevated plasma coagulation factor VIII has been considered a factor related to recurrent venous thromboembolism. Here we report a patient who had recurrent CVT associated with significantly elevated levels of factor VIII despite the chronic use of an antiplatelet agent. Factor IX was also elevated in this patient. These findings suggest that elevated factor VIII is a factor underlying the recurrence of CVT, and that prolonged anticoagulation therapy may have to be considered in patients with elevated coagulation factor levels.
\end{abstract}

J Clin Neurol 2(4):286-289, 2006

Key Words : Cerebral venous sinus thrombosis, Coagulation factor VIII

\section{INTRODUCTION}

Cerebral venous thrombosis (CVT) is an uncommon condition with a variety of clinical presentations and outcomes, ${ }^{1}$ and hence definitive therapeutic and prevention strategies for CVT have not been established. ${ }^{1,2}$ Although anticoagulation therapy is used commonly in patients with CVT, a decision to initiate, continue, or withhold anticoagulation therapy in these patients should be made on the basis of the anticipated risk of recurrent thrombosis and possible hemorrhagic complications. Although it is known that CVT can be caused by pregnancy, hypercoagulable condition, inflammatory states, or collagen vascular diseases ${ }^{1,3}$, the factors related to recurrent CVT remain unclear. Recent studies have addressed the hypothesis that elevated plasma factor VIII is an independent risk factor for recurrent venous thromboembolism. ${ }^{4-6}$ However, there have been few reported cases of recurrent CVT with elevated plasma factor VIII, and to our knowledge no Korean cases have been described. To address this issue, here we report a patient who had recurrent episodes of CVT with elevated factor VIII despite prolonged use of an antiplatelet agent.

\section{CASE REPORT}

A 46-year-old woman developed severe generalized headache and vomiting. Sudden right postauricular area pain developed 10 days before admission, which spread

Received : October 18, 2006 / Accepted : November 20, 2006 / Address for correspondence : Jong S. Kim, M.D.

Department of Neurology, Asan Medical Center, University of Ulsan College of Medicine, 388-1 Pungnap-dong, Songpa-gu, Seoul 138-736, Korea Tel: +82-2-3010-3442, Fax: +82-2-4744691, E-mail: jongskim@amc.seoul.kr

* This study was supported by a research grant from the Korean Ministry of Health and Welfare (no. 03-PJ1- PG1-CH06-0001) and a grant from the Brain Research Center of the 21st Century Frontier Research Program, which is funded by the Ministry of Science and Technology of Korea (no. M103KV010010 06K2201 01010). 
to the frontal area. The headache was severe, and dull or throbbing in nature. She also complained of blurred vision at the time of admission. This patient did not have hypertension or diabetes mellitus, and she was a nonsmoker. She denied a history of orogenital ulcer, ocular diseases, oral contraceptive use, or regular ingestion of alcohol or drugs. On neurologic examination, she was alert and oriented. She showed no focal neurologic findings except for bilateral papilledema.

Four years before this admission, she had developed generalized tonic-clonic seizures and, based on angiographic findings, was diagnosed as having superior sagittal sinus thrombosis. Cerebral venous infarction was found in the left frontal area (Fig. 1). At that time, her serum level of factor VIII was $216 \%$ (normal range $60-120 \%$ ) and that of factor IX was 238\% (normal range $60-120 \%$ ). Plasma levels of fibrinogen, C-reactive
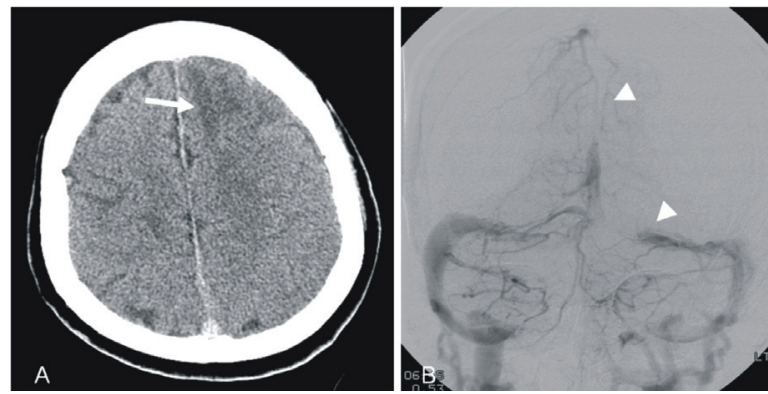

Figure 1. Brain computed tomography (CT) scan and conventional angiogram acquired at the time of the first attack. (A) CT scan showing a low-density area on the left frontal area (arrow). (B) Angiography revealed a filling defect in the superior sagittal sinus and irregular filling in the left transverse sinus (arrowheads).
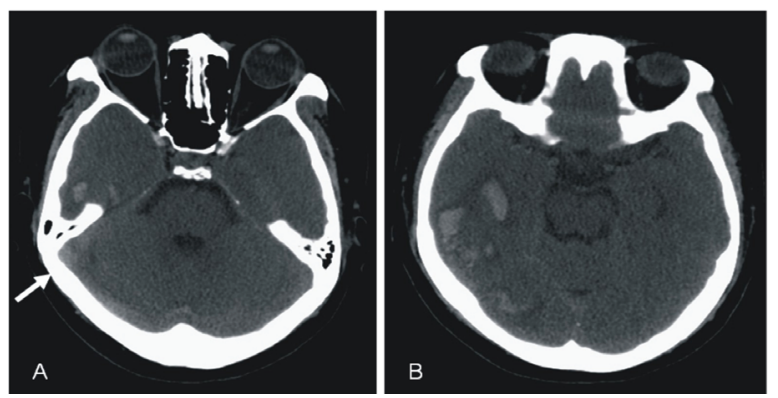

Figure 2. CT findings at the time of the second attack. (A) Transverse sinus thrombosis with cord sign is visible on the right side (arrow). (B) CT showing venous infarction with hemorrhagic transformation in the right temporal area. protein, total protein $\mathrm{S}$, protein $\mathrm{C}$, and homocysteine were normal. She was negative for lupus anticoagulant, and only weakly positive for anticardiolipin antibody of IgM type. She was treated at that time with anticoagulation therapy for 5 months, and then maintained with continuous antiplatelet (ticlopidine) therapy. Her serum levels of coagulation factor were not measured during the subsequent follow-up period.

On admission, a brain computed tomography scan showed hemorrhagic infarction in the right temporal area. High-density signals in the right jugular vein and transverse sinus suggested acute venous thrombosis (Fig. 2). Venous infarction with hemorrhagic transformation was diagnosed. The laboratory results revealed a threefold elevation of plasma factor VIII (339\%). Factor IX was also mildly elevated (151\%). Her white blood cell count and hemoglobin levels were normal. Other laboratory results, such as C-reactive protein, fibrinogen, protein $\mathrm{C}$, total protein $\mathrm{S}$, and homocysteine, were within normal limits. We was negative for lupus anticoagulant, and only weakly positive for beta-2glycoprotein of I-IgM type, and her thyroid functions including free thyroxin and thyroid-stimulating hormone were within normal ranges. Her blood type was A.

Multiple venous sinus thromboses were found on conventional angiography, and suction thrombectomy was performed on the right internal jugular vein (Fig. 3). Low-molecular-weight heparin (LMWH) and mannitol were administered. Her headache gradually improved and the papilledema disappeared after several days. Subcutaneous LMWH was switched to warfarin at the
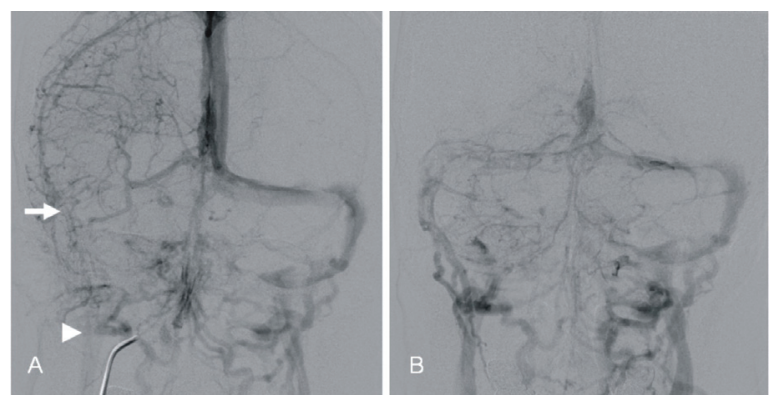

Figure 3. Conventional angiogram findings. (A) Occlusion of the right jugular vein (arrowhead) and transverse sinus (arrow). (B) After suction thrombectomy, the filling of the right internal jugular vein and transverse sinus had improved. 
time of discharge, and the target International Normalized Ratio range of 2-3 was maintained. At a 1-month follow-up her course remained uneventful.

\section{DISCUSSION}

Our patient had recurrent CVT despite the continuous use of an antiplatelet agent. Recurrent CVT is uncommon, ${ }^{1,2}$ with an approximate annual rate of $5 \%{ }^{2}$ It has been suggested that the concurrent presence of cancer, high titer of antiphospholipid antibodies, or chronic inflammation are factors related to the recurrence of CVT. ${ }^{1,3,7}$ Our patient did not have these risk factors, but showed significantly elevated plasma levels of factor VIII and moderately elevated plasma levels of factor IX.

Recently it was proposed that a high plasma level of factor VIII could be a risk factor for recurrent venous thrombosis. ${ }^{4}$ In the Leiden Thrombophilia Study, factor VIII levels above $150 \mathrm{IU} / \mathrm{dl}(\%)$ were associated with a fivefold increased risk of venous thrombosis. ${ }^{8}$ Among patients with a factor VIII level above the 90th percentile, the likelihood of recurrence at 2 years was $37 \%$, as compared with a $5 \%$ likelihood among patients with lower levels. ${ }^{4}$ Although not yet well recognized, elevated plasma factor VIII levels may not only be a factor related to the recurrence of CVT, but may itself also be a risk factor for CVT. In a recent study, elevated plasma factor VIII was considered to be the most important prothrombotic risk factor for CVT. ${ }^{7}$

One may argue that these elevated clotting factors may represent an acute-phase reaction rather than the cause of the thrombosis. Unfortunately, plasma factor VIII was not measured in the chronic stage of disease in our patient. However, previous literature has illustrated that the high levels of factor VIII in patients with thrombosis persist over time. ${ }^{4,59}$ Moreover, as in our case, a significant relationship was not detected between plasma levels of factor VIII and those of C-reactive protein. ${ }^{4,9}$ Finally, although plasma levels of factor VIII may increase in patients with inflammation, cancer, or pregnancy, ${ }^{1}$ our patient did not have these factors. The high levels of factor VIII can therefore be considered the cause rather than the consequence of venous thrombosis.
The etiology underlying the high plasma factor VIII levels remains unclear. An elevated von Willebrand factor and a non-O blood group have been considered important determinants of plasma factor VIII levels. ${ }^{10,11}$ In another study, a gene locus on chromosome 18 was shown to influence both factor VIII levels and susceptibility for thrombosis. ${ }^{12}$ Therefore, certain genetic factors appear to predispose a subject to have elevated plasma factor VIII and recurrent thrombosis.

Interestingly, plasma factor IX was also elevated in our patient. According to a recent report, a high plasma factor IX level may also be related to a two- to threefold increased risk of venous thrombosis. ${ }^{13}$ Since factor VIII is the cofactor of factor IXa in the activation of factor $\mathrm{X}$, the concurrent increase in plasma factors VIII and IX may further increase the risk of CVT. However, the mechanism underlying the concomitant increase in these two coagulation factors remains unknown.

The treatment of CVT remains controversial. We successfully performed suction thrombectomy through the internal jugular vein in our patient. This treatment has been tried in the few reported cases, ${ }^{14}$ but its efficacy has never been examined in controlled studies. Trials with heparin and other anticoagulants have not proved beneficial; ${ }^{1}$ nevertheless, anticoagulants have been considered a standard therapy for CVT. Because of the risk of bleeding, however, long-term anticoagulant therapy is generally reserved for patients with a high risk of recurrent CVT.

Considering the variable clinical course of CVT, we cannot draw any definitive conclusion from our single case. Nevertheless, our findings in this patient suggest that antiplatelet therapy is not sufficient to prevent recurrent CVT in patients with significantly elevated coagulation factors. Factor VIII, and perhaps factor IX, may have to be routinely screened in patients with CVT, and prolonged anticoagulation therapy may have to be considered in those with elevated levels of these factors.

\section{REFERENCES}

1. Stam J. Thrombosis of the cerebral veins and sinuses. $N$ Engl J Med 2005;352:1791-1798. 
2. Schulman S, Rhedin AS, Lindmarker P, Carlsson A, Larfars G, Nicol P, et al. A comparison of six weeks with six months of oral anticoagulant therapy after a first episode of venous thromboembolism. Duration of Anticoagulation Trial Study Group. N Engl J Med 1995;332: 1661-1665.

3. Sebire G, Tabarki B, Saunders DE, Lerov I, Leisner R, Saint-Martin C, et al. Cerebral venous sinus thrombosis in children: risk factors, presentation, diagnosis and outcome. Brain 2005;128:477-489.

4. Kyrle PA,Minar E, Hirschl M, Bialonczyk C, Stain M, Schneider B, et al. High plasma levels of factor VIII and the risk of recurrent venous thromboembolism. $N$ Engl $J$ Med 2000;343:457-462.

5. O’Donnell J, Mumford AD, Manning RA, Laffan M. Elevation of FVIII:C in venous thromboembolism is persistent and independent of the acute phase response. Thromb Haemost 2000;83:10-13.

6. Kraaijenhagen RA, in't Anker PS, Koopman MM, Reitsma $\mathrm{PH}$, Prins $\mathrm{MH}$, van den Ende A, et al. High plasma concentration of factor VIII is a major risk factor for venous thromboembolism. Thromb Haemost 2000;83:5-9.

7. Pierangeli SS, Chen PP, Gonzalez EB. Antiphospholipid antibodies and the antiphospholipid syndrome: an update on treatment and pathogenic mechanisms. Curr Opin Hematol 2006;13:366-375.

8. Cakmak S, Derex L, Berruyer M, Nighoghossian N, Philippeau F, Adeleine $\mathrm{P}$, et al. Cerebral venous thrombosis: clinical outcome and systemic screening of prothrombotic factors. Neurology 2003;60:1175-1178.

9. Kamphuisen PW, Eikenboom JC, vos HL, Pablo R, Sturk A, Bertina RM, et al. Increased levels of factor VIII and fibrinogen in patients with venous thrombosis are not caused by acute phase reactions. Thromb Haemost 1999;81:680-683.

10. Koster $\mathrm{T}$, Blann $\mathrm{AD}$, Briet E, Vandenbroucke JP, Rosendaal FR. Role of clotting factor VIII in effect of von Willebrand factor on occurrence of deep-vein thrombosis. Lancet 1995;345:152-155.

11. Gill JC, Endres-Brooks J, Bauer PJ, Marks WJ Jr, Montgomery RR. The effect of ABO blood group on the diagnosis of von Willebrand disease. Blood 1987;69:16911695.

12. Soria JM, Almasy L, Souto JC, Buil A, Martinez-Sanchez $\mathrm{E}$, Mateo J, et al. A new locus on chromosome 18 that influences normal variation in activated protein $\mathrm{C}$ resistance phenotype and factor VIII activity and its relation to thrombosis susceptibility. Blood 2003;101:163167.

13. van Hylckama Vlieg A, van der Linden IK, Bertina RM, Rosendaal FR. High levelsof factor IX increase the risk of venous thrombosis. Blood 2000;95:3678-3682.

14. Tajima H, Murata S, Kumazaki T, Ichikawa K, Tajiri T, Yamamoto Y. Successful interventional treatment of acute internal jugular vein thrombosis. AJR Am J Roentgenol 2004;182:467-469. 